\title{
Practical Implementation of the Spatial Images Technique for the Analysis of Shielded Multilayered Printed Circuits
}

\author{
Juan Sebastian Gómez-Díaz, Student Member, IEEE, Monica Martínez-Mendoza, Student Member, IEEE, \\ Francisco Javier Pérez-Soler, Student Member, IEEE, Fernando Quesada-Pereira, Member, IEEE, and \\ Alejandro Alvarez-Melcón, Senior Member, IEEE
}

\begin{abstract}
In this paper, a practical implementation of the spatial images technique for the analysis of shielded multilayered printed circuits inside convex cavities is proposed. A new method is introduced in order to automatically locate the images surrounding the structure in order to impose the appropriate boundary conditions for the potentials. The boundary conditions are imposed at discrete points along the cavity wall and, therefore, the technique proposed is an approximation to the exact cavity modeling. Furthermore, for the analysis of electrically long cavities, the use of several rings of images surrounding the entire cavity at different heights is employed. Using the special features of the formulation, a new method of moments implementation combined with the spatial images technique is proposed in order to efficiently analyze practical multilayered printed filters, considerably reducing the computational cost. Several examples with CPU time comparisons are provided, demonstrating the accuracy and efficiency of the new technique. A novel transversal filter in a trapezium-shaped cavity is designed, manufactured, and tested for the first time using the spatial images technique.
\end{abstract}

Index Terms-Boxed circuits, cavities, Green's functions, integral equations (IEs), printed circuits, spatial images, transversal filters.

\section{INTRODUCTION}

$\mathbf{T}$ ODAY, the accurate analysis and design of modern microwave systems is fundamental due to their wide use in telecommunication applications. Complex circuits with high integration degree, operating at high frequencies, and with metallic walls providing shielding to the structures, must be rapidly analyzed. An interesting possibility to perform this kind of analysis is to employ the integral equation (IE) technique combined with the method of moments (MoM) [1]. In this case, the Green's functions associated with the multilayered shielded medium must be calculated.

A new spatial-domain numerical method for the Green's functions computation in circular cylindrical cavities was presented in [2]. The method was modified in order to analyze complex-shaped cylindrical cavities, defined with linear segments, in [3]. The idea of the method is to use charge and dipole images outside the cavity to enforce the proper boundary conditions for the potentials. The boundary conditions are imposed at discrete points along the cavity wall, and thus, the technique proposed should be considered as an approximation to the real cavity modeling. To do that, the free-space Green's function was employed in [3]. In this paper, we incorporate the multilayered media Green's functions formulated as Sommerfeld integrals [4] in order to study real closed multilayered cavities including top and bottom covers. This idea was first proposed in [2] in the context of circular cylindrical cavities. In this paper, we combine the same idea with the formulation presented in [3] to analyze circuits printed in convex cavities.

In all the previous studies, the location of the spatial images around the cavity was taken as an additional degree of freedom. Generally speaking, the spatial images were located at a given fixed distance from the wall, following the shape of the cavity. However, the exact location of the spatial images might affect the accuracy and numerical stability of the technique, especially when the source point is close to the walls of the cavity. Although a specific spatial images distribution could provide good accuracy when it is used with a particular type of cavity and source position, the same distribution also might not be able to achieve the same accuracy for another cavity shape or when the source is placed at a different location. Therefore, a systematic technique for the placement of the spatial images must be devised in order to preserve accuracy for all source points locations and different cavity shapes.

The second difficulty of the spatial images technique is that the boundary conditions for the fields are imposed at only one transverse plane along the height of the cavity. Consequently, for electrically long cavities, the behavior of the fields along the cavity height might degrade. For this reason, it is important to extend the original formulation so that electrically long cavities can be analyzed without loss of precision. The basic idea is to also impose the boundary conditions at discrete points along the cavity height using several rings of images. This technique is also an approximation to the real behavior of the field along the cavity height. However, it will be shown in this paper that the error of the fields along the cavity height can be made small if a sufficient number of rings are selected. 
Finally, a practical limitation of the spatial images method is its computational cost when it is used inside IE formulations for the analysis of practical shielded circuits. This is due to the necessity to calculate exact charge and dipole images values for every new source point location. These values are calculated through the solution of linear systems. Although the sizes of the systems are, in general, small, they have to be calculated many times during the MoM implementation. This makes the original technique computationally very intensive.

In this paper, specific solutions are proposed for the abovementioned problems. It is shown that when using the new proposed techniques, the efficient analysis of practical multilayered shielded circuits employing the spatial images method is possible. First, a procedure to automatically place the spatial images outside a given cavity is proposed. This distribution depends on the shape of the cavity and on the source position, providing acceptable accuracy in all situations. Second, a multiring images scheme is proposed in order to analyze electrically long structures by sampling the entire cavity at different discrete heights. We show that with this technique, the accuracy in the imposition of the boundary conditions for the fields can be maintained along the entire cavity height. Finally, a new MoM implementation is presented in order to increase efficiency using the special features of the method. The key point of the new technique is the natural separation of the Green's functions in two parts, namely, the source and image contributions. The singularity of the Green's function is present only in the source term, and can be computed very fast using standard techniques for Sommerfeld transformations [5]. The systems of linear equations need to be computed only for the contribution of the spatial images. However, the images contribution exhibits a very smooth behavior, and it can be treated with less numerical effort during the MoM solution.

Finally, three practical microwave filters are analyzed and their CPU times are compared to other methods. The first circuit is a square-encapsulated bandpass printed filter based on coupled-line sections. The second circuit uses a novel hybrid technology reported in [6] to implement a second-order transversal filter inside a trapezium-shaped cavity. To the authors knowledge, this is the first time that a microwave filter is designed, manufactured, and tested in a trapezium-shaped cavity using the spatial images Green's functions inside IE formulations. This example also shows the ability of the new formulation for the design of practical filters in complex shaped cavities. The third and last circuit is a four-pole broadside-coupled filter. The circuits responses have been validated with measured data. The agreement obtained with the novel theory is good in all cases with high computational performance, as compared to other techniques.

\section{THEORETICAL OVERVIEW}

The idea of the spatial images technique is to impose the boundary conditions for the potentials at $N$-discrete points along the cavity wall using $N$-images placed outside the structure. The technique can be seen, therefore, as an approximation to the real behavior of the fields along the cavity wall of the structure. The strength and orientation of the images are then computed so the proper boundary conditions are satisfied at

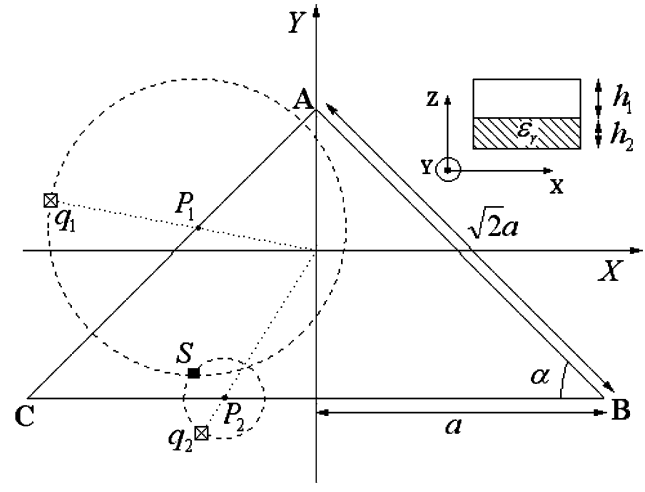

Fig. 1. Example of the image distribution algorithm. $S$ is the source position, $P_{1}$ and $P_{2}$ are two points at the cavity wall, and $q_{1}$ and $q_{2}$ are the two images positions. $a=\lambda, h_{1}=0.2 \lambda, h_{2}=0.1 \lambda$, and $\epsilon_{r}=5.0$.

discrete points of the metallic wall. The details of the formulation for arbitrarily shaped cavities employing free-space Green's functions can be found in [3]. However, for the analysis of completely closed enclosures, the bottom and top covers of the cavity must be included in the formulation. The most efficient way to accomplish this task is to formulate the Green's functions of a multilayered medium in the spatial domain using the Sommerfeld transformation. In this way, the presence of dielectric layers inside the cavity can also be automatically accounted for. Note that the method proposed imposes the boundary conditions for the fields considering perfect electric cavity walls. In this way, the losses due to the finite conductivity of the cavity walls cannot be easily modeled with this formulation. However, the losses in the dielectric substrates are easily included using the spatial-domain multilayered Green's functions formulated as Sommerfeld integrals [4]. The losses in the printed metallizations are also easily included using the Leontovich boundary condition combined with the concept of the surface impedance of a nonperfect conductor [7].

In this paper, we have combined the original technique described in [3] with the multilayered media Green's functions in the spatial domain formulated through the Sommerfeld transformation. This has allowed us to study real multilayered printed circuits shielded in cavities of complex shapes. In Sections II-A-C, we present several techniques allowing a practical implementation of the spatial images formulation for the analysis of real microwave shielded components.

\section{A. Spatial Images Distribution}

The distribution of the images around the cavity is essential in the spatial images technique in order to obtain accurate results. A triangular cylindrical cavity is depicted in Fig. 1 in order to introduce a new algorithm to automatically place the spatial images around a given cavity.

The first step of the method consists of sampling the cavity contour at $N$-discrete points. Each of these points will be the center of a virtual circle with radius equal to the distance to the source position inside the cavity (see Fig. 1). To locate each single spatial image, a line is traced from the center of the waveguide to each discrete point at the wall. These lines will intersect the virtual circles at a maximum of two points. The position of the image is selected at the intersecting point falling outside of the cavity. An example of the algorithm for two different points 


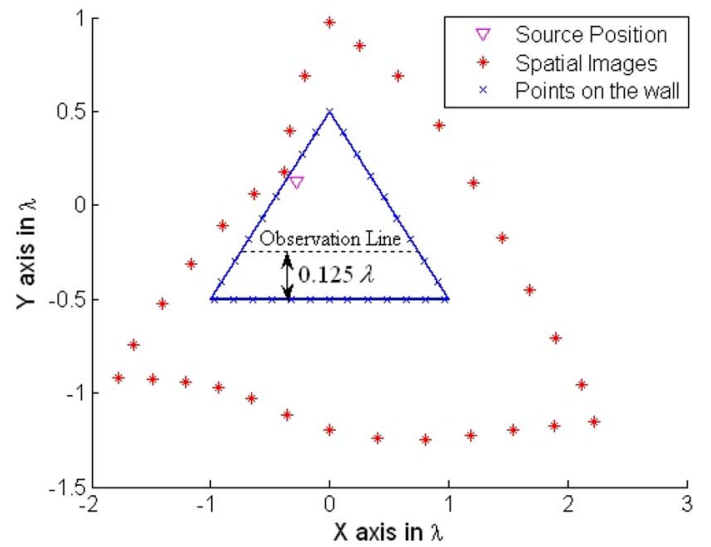

Fig. 2. Images arrangement around the triangular cavity depicted in Fig. 1 when the source is placed at the position $(-0.30 \lambda, 0.13 \lambda, 0.1 \lambda)$.

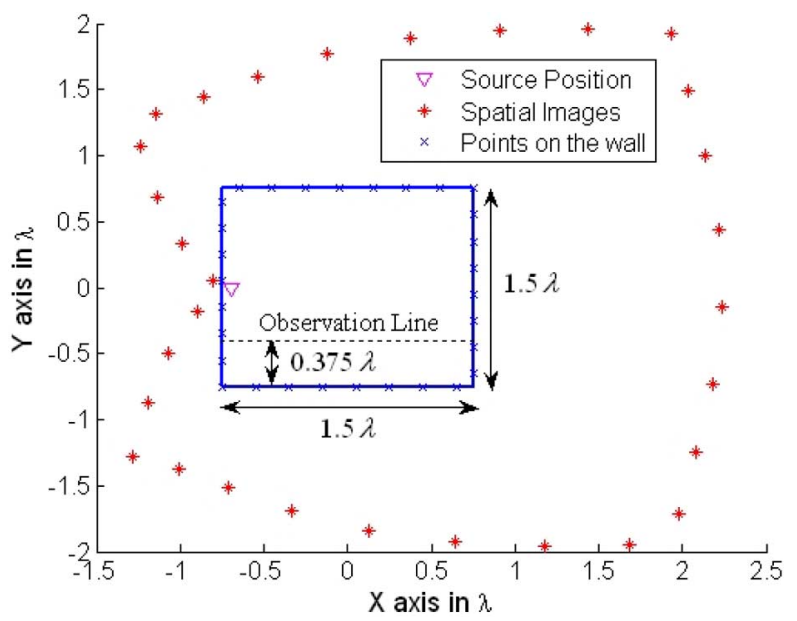

Fig. 3. Images arrangement around a square cavity when the source is placed at the position $(-0.74 \lambda, 0.0 \lambda, 0.1 \lambda)$. The substrate arrangement is shown in Fig. 1.

$P_{1}$ and $P_{2}$ is shown in Fig. 1. It is important to note that when the source is placed near a cavity wall, the method locates one image close to that wall. We have observed that this situation leads to increased accuracy for source points close to the wall. An example of this situation is presented in Fig. 2 where we show the final location of the images, obtained with this algorithm, for a situation of a source point placed very close to a wall. To show how the algorithm behaves for a square cavity, we further present in Fig. 3 the final location of the images when the source point is placed at the position $(-0.74 \lambda, 0.0 \lambda, 0.1 \lambda)$. In both cases, we observe that one spatial image approaches the source point from outside the cavity. This behavior correctly simulates the situation of a source point very close to an infinite ground plane. By image theory, we know that, in this case, the spatial image must be placed at the same distance from the ground plane as the source [8]. This behavior of the basic image theory is respected by the new algorithm, leading to an increased accuracy for points close to the cavity walls.

To demonstrate the usefulness of the technique, we present in Fig. 4 the magnetic vector potential $G_{A}^{y y}$-component along the observation line shown in Fig. 3 when the source point is placed at the center of the cavity. We compare the results obtained using

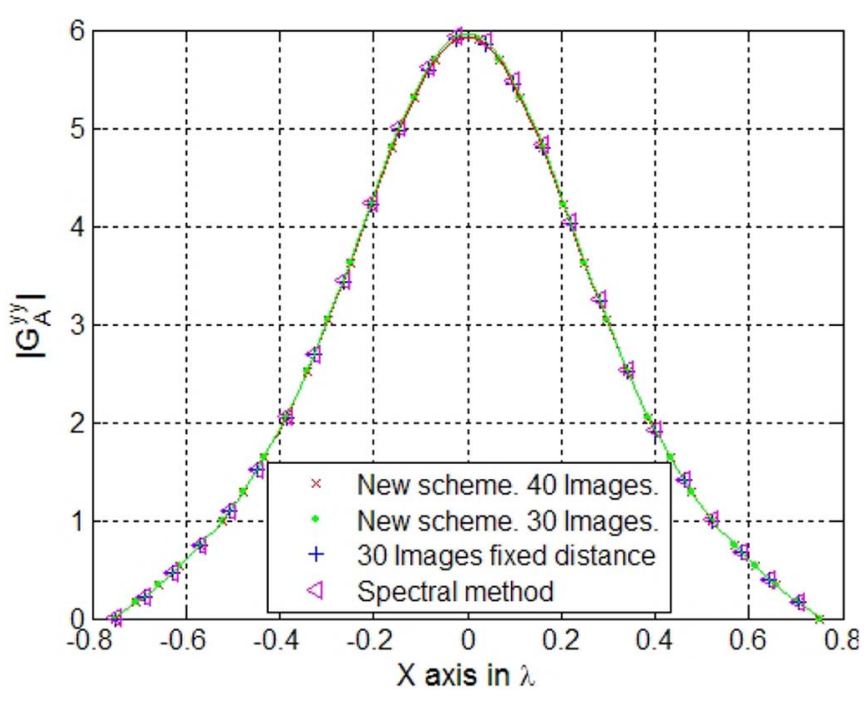

Fig. 4. Magnetic vector potential $\left|G_{A}^{y y}\right|$-component along the observation line of Fig. 3. The source is placed at the center of the cavity.

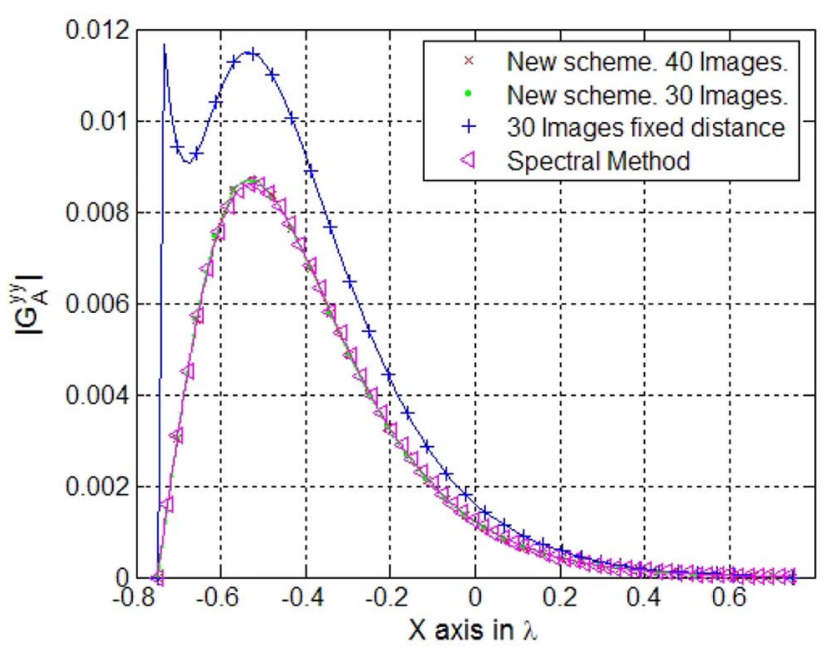

Fig. 5. Magnetic vector potential $\left|G_{A}^{y y}\right|$-component along the observation line of Fig. 3. The source is placed at the position $(-0.74 \lambda, 0.0 \lambda, 0.1 \lambda)$.

the new algorithm with the results obtained when the spatial images are placed following the cavity contour at a fixed distance of $0.5 \lambda$ from the wall. For validation, the results obtained with a sepctral-domain approach, only valid for rectangular cavities, are also included [9]. Finally, to show the convergence of the method, the results obtained using the new algorithm are presented for two different number of images (30 and 40 images around the cavity contour). We observe in Fig. 4 that the results are very similar in all cases (with maximum relative errors below $0.08 \%$ ), showing that convergence has been achieved.

The test just performed shows the numerical stability of the spatial images technique when the source is placed at the center of the cavity. We have repeated a similar study in Fig. 5, but when the source point is close to the left wall of the cavity $(-0.74 \lambda, 0.0 \lambda, 0.1 \lambda)$. In this case, the results obtained with the new algorithm with 30 and 40 images agree very well with the sepctral-domain technique (the maximum relative error is below $0.05 \%)$. However, the results obtained placing the images at a fixed distance from the cavity wall fail to converge. This test 


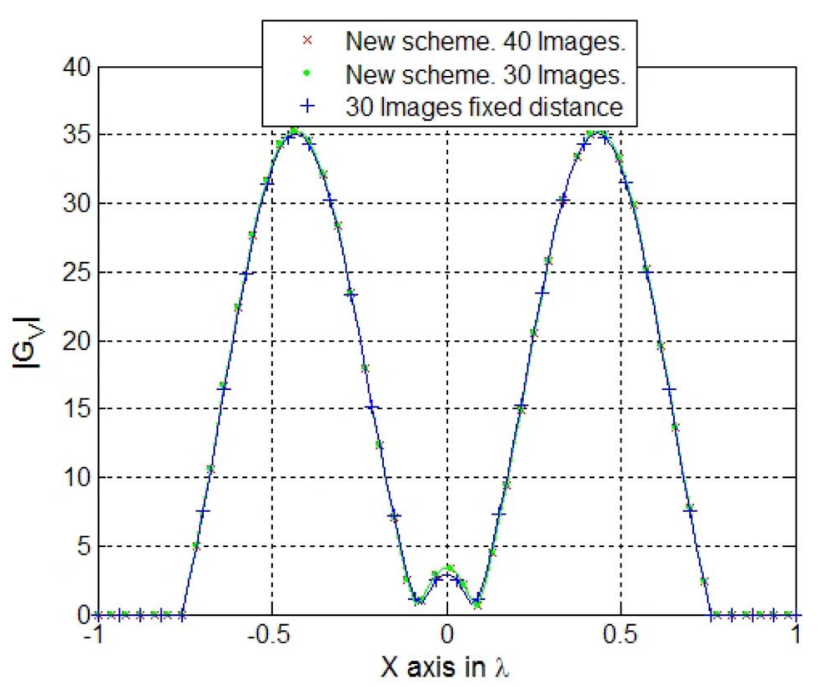

Fig. 6. Electric scalar potential $\left|G_{V}\right|$ along the observation line of Fig. 2. The source is placed at the cavity center.

shows that the location of the spatial images is very important when the source point is close to the cavity walls. It also shows the high robustness of the algorithm proposed for the placement of the images, even for source points very close to the walls.

To further demonstrate how the algorithm behaves in a cavity of different shape, we present a similar test in the triangular cavity of Fig. 2. In this case, we present in Fig. 6 the Green's function for the electric scalar potential along the observation line shown in Fig. 2, when the source point is placed again at the center of the cavity. We have compared the results obtained using the new algorithm with 30 and 40 images with the results obtained when the spatial images are located around the cavity at a fixed distance of $0.5 \lambda$. In this case, all results agree well (the maximum relative error is below $0.5 \%$ ), showing the stability of the spatial images technique. Next, we present in Fig. 7 similar results, but when the source point is placed very close to one of the cavity walls $(-0.30 \lambda, 0.13 \lambda, 0.1 \lambda)$. In this case, the results obtained with the new algorithm for 30 and 40 images agree well, indicating that convergence has been reached (the maximum relative error is below $0.09 \%$ ). However, the results obtained when the images are placed at a fixed distance from the cavity are different, showing a lower convergence rate.

To give an explanation of the lower convergence rate when the source is close to the cavity walls, we present in Fig. 8 the electric scalar potential around the triangular cavity contour. For this test, the source point is again placed close to one of the walls $(-0.30 \lambda, 0.13 \lambda, 0.1 \lambda)$. Ideally, the electric scalar potential should always be zero around the cavity wall. The dashed line of Fig. 8 shows that the potential is essentially zero, except in the region corresponding to the wall close to the source point. The proximity of the source point to this wall produces abrupt variations of the potential in this area. In this same figure, we denote via a thick line the potential obtained when the new algorithm is employed to locate the spatial images. We can observe that, in this case, the potential around the cavity wall is very small, even along the wall close to the source point. The improvement obtained for the potential in this area is clearly observable in the results of Fig. 8. This last result confirms that a

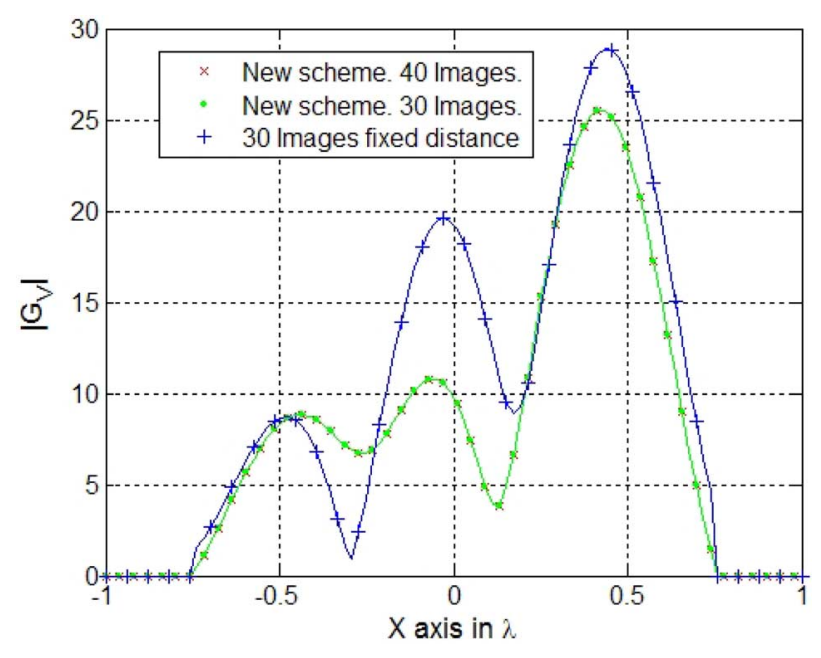

Fig. 7. Electric scalar potential $\left|G_{V}\right|$ along the observation line of Fig. 2. The source is placed at the position $(-0.13 \lambda, 0.13 \lambda, 0.1 \lambda)$.

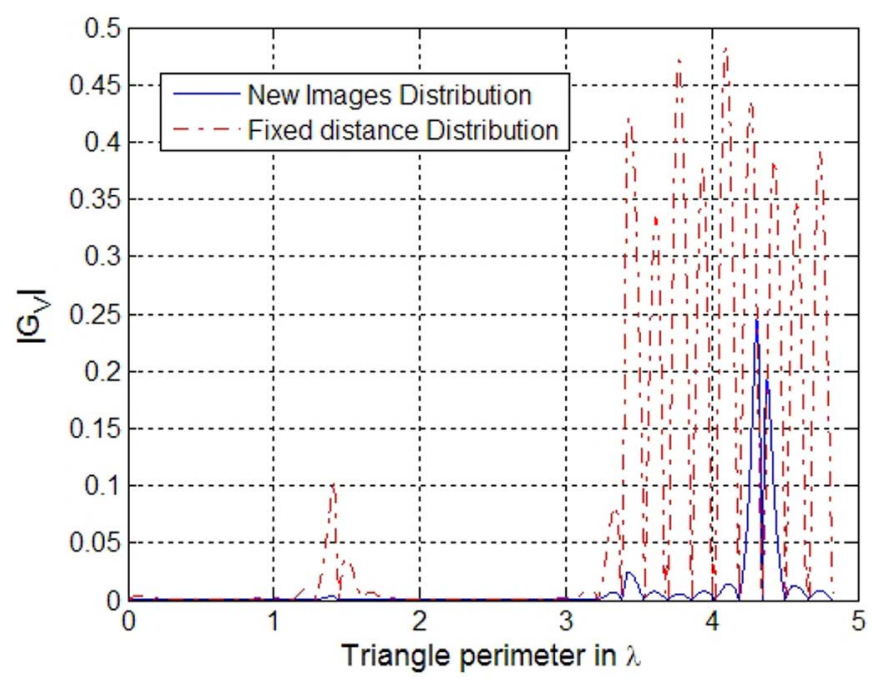

Fig. 8. Electric scalar potential $\left|G_{V}\right|$ along the triangular cavity contour (see Fig. 1). The source is placed close to the wall at the position $(-0.13 \lambda, 0.13 \lambda, 0.1 \lambda)$.

clever selection for the location of the images around the cavity leads to an improved convergence and numerical precision in the imposition of the relevant boundary conditions.

There is a physical explanation for the improved accuracy when the new technique to locate the images is used. When the source is close to a wall, the relevant geometrical detail influencing the behavior of the Green's functions is the wall close to the source, which tends to behave as an infinite ground plane, as the source approaches the wall. Using the proposed technique to locate the images, one of the spatial images will be placed in a mirror position to the source point with respect to this wall, which is in agreement with the spatial images solution for an infinite ground plane [8].

The importance of the algorithm introduced is twofold. First, the algorithm places the spatial images in an automatic fashion for any convex shape of the cavity considered. Second, the accuracy and stability of the algorithm drastically increases for source points very close to the walls, as we have just discussed. 


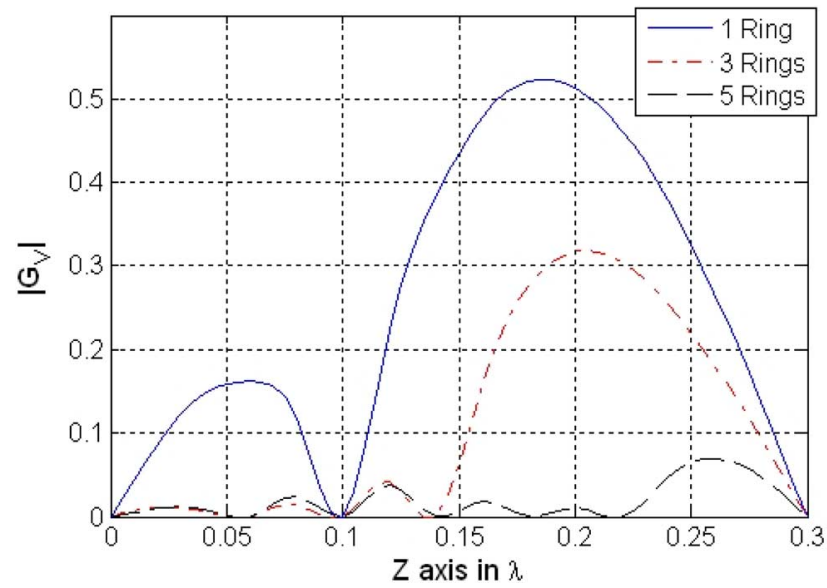

Fig. 9. Electric scalar potential $\left|G_{V}\right|$ along the $Z$ axis of the cavity depicted in Fig. 1 analyzed with one, three, and five rings.

\section{B. Multiring Rearrangement}

Initially, the spatial images method employs $N$ images to impose the boundary conditions at $N$ discrete points situated at a given cross section of the cavity. However, if the height of the cavity is electrically large, the imposition of the boundary conditions in one cross section of the cavity might not suffice to represent the correct behavior of the fields along the entire height. What we propose is to use $M$ rings, each one having $N$ images, to impose the boundary conditions at several discrete heights of the cavity. In this case, $(M \cdot N)$ total boundary conditions are imposed on the cavity wall. To show the effectiveness of the idea proposed, we present in Fig. 9 the electric scalar potential at the waveguide wall, plotted along the height of the triangular cavity introduced in Fig. 1. The results are given when one, three, and five rings of images are included in the calculations. Ideally, the electric scalar potential must be zero at all points of the cavity wall to respect the boundary conditions. Via the solid line, we include the results when only one ring of images is placed at $z=0.1 \lambda$. We observe that the potential is zero at one point, but the amplitude rapidly grows along the height of the cavity. Via the dashed-dotted line, we show the results obtained when three rings of images, placed at heights $z=0.06 \lambda, 0.1 \lambda, 0.14 \lambda$, are included. We observe that the value of the potential along the substrate height is now very small (from $z=0$ to $z=0.1 \lambda$ ). However, in the air region, the potential still grows to unacceptable values. Finally, if we place five rings of images scattered along the cavity height at positions $z=0.06 \lambda, 0.1 \lambda, 0.14 \lambda, 0.18 \lambda, 0.22 \lambda$, the value of the potential remains very low along the entire cavity height. This demonstrates that the boundary conditions can be maintained within a given accuracy, and demonstrates the usefulness of the multiring approach to model electrically long cavities.

From a practical point of view, it is interesting to establish a strategy to place the rings of images. In general, a ring can be placed at each interface where the microwave circuits are printed. In this way, the error in the fulfillment of the boundary conditions for the fields at these interfaces will be minimum (they will correspond to the zeros shown in Fig. 9). Moreover, the electrical length of the cavity height is fundamental to decide the number of rings to employ. We have observed that a new ring must be placed for each $\lambda / 3$ of the cavity height. Furthermore, the presence of dielectric layers can excite new resonant modes. In this case, additional rings can be added to properly model these modes.

\section{Efficient Novel MoM Implementation}

The technique developed for the Green's functions calculation can be used for the analysis of practical printed circuits inside arbitrarily shaped cylindrical enclosures. To do that, the IE technique combined with the MoM [1] is employed. The printed circuit is discretized using subdomain basis functions [10] and they are combined with efficient integration techniques [11] for the calculation of the MoM matrix. A Galerkin procedure is used to solve the final IE. Since the enclosure and dielectric layers are already taken into account by the formulated Green's functions, only the printed metallizations of the circuit need to be considered during the MoM solution. However, a direct application of the MoM procedure to the circuit using the new Green's functions will be too time consuming. This is because if $N$ images are employed, one system of linear equation with $N$ unknowns (for the electric scalar potential) and two systems with $2 N$ unknowns (for the magnetic vector potential) must be solved for any combinations of source and observation points.

To circumvent this difficulty, a new IE implementation is proposed based on the special features of the new formulation. One of the important properties of the spatial images formulation is that the source term is naturally separated from the contribution of the images that takes into account the effects of the cavity lateral walls. Using this important feature, the total boxed Green's functions can be naturally expressed in two terms as

$$
\overline{\bar{G}}_{T}\left(\vec{r}, \vec{r}_{n}^{\prime}\right)=\overline{\bar{G}}_{\text {Source }}\left(\vec{r}, \vec{r}_{n}^{\prime}\right)+\overline{\bar{G}}_{\text {Images }}\left(\vec{r}, \vec{r}_{n}^{\prime}\right)
$$

where $\overrightarrow{r_{n}^{\prime}}$ is the position vector of the source point and $\vec{r}$ denotes the position of the observation point. The source contribution has a strong singularity involving fast variations. This is demonstrated in Fig. 10 where we present the $G_{A}^{y y}$ source contribution to the Green's function inside the triangular cavity shown in Fig. 1. We clearly observe a strong peak in the potential due to the source located at the center of the cavity. The singular behavior of the source directly depends on the Sommerfeld transformation [12] for a particular layered structure. To integrate this term, the singularity must be properly handled. However, this term can be computed very fast using standard numerical techniques available for the efficient evaluation of Sommerfeld integrals [5].

On the other hand, the second term in (1) corresponds to the contribution of the images. It is the evaluation of this term that requires the solution of the systems of linear equations. Fortunately, all images are located outside of the cavity so they do not contain inside any singular behavior. Consequently, this term exhibits very smooth variations inside the cavity. This is demonstrated in Fig. 11 where we present the same Green's function component as before, but now only the contribution of the images is included. Due to this smooth behavior, this term can be easily integrated with limited computational effort.

Using these features, two MoM matrixes are computed separately. The first one $\left(Z_{\text {Source }}\right)$ contains the singular behavior 


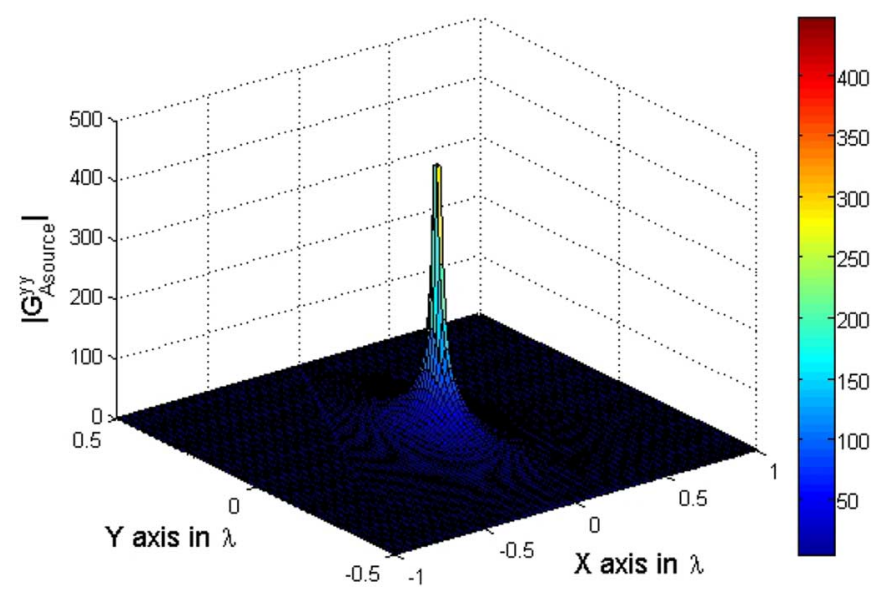

Fig. 10. Contribution of the source for the $G_{A}^{y y}$ component of the magnetic dyadic Green's function at a middle height in the cavity shown in Fig. 1. The source is placed at the center of the cavity $(0,0,0.1 \lambda)$.

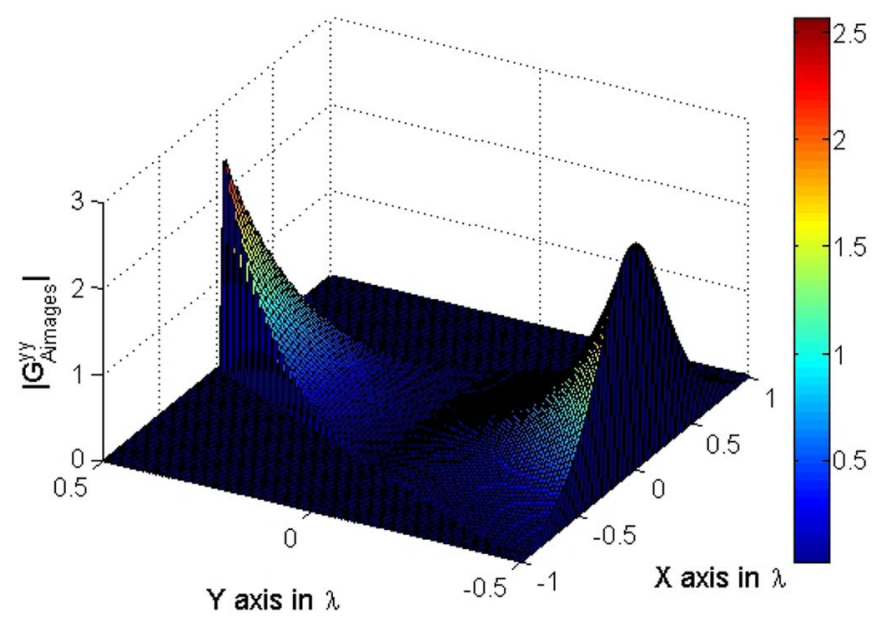

Fig. 11. Contribution of the images for the $G_{A}^{y y}$ component of the magnetic dyadic Green's function at a middle height in the cavity shown in Fig. 1. The source is placed at the center of the cavity $(0,0,0.1 \lambda)$.

of the Green's functions and can be evaluated fast using efficient numerical techniques for the computation of the Sommerfeld transformation. The second one $\left(Z_{\text {Images }}\right)$ contains the contribution of the images, and due to the smooth behavior observed, it can be computed with limited computational effort. There is also no singularity in this term since the images are located outside of the cavity region. Due to these properties, we have observed that, in most cases, the MoM matrix associated to this term can be integrated using only one point in each of the discretization cells used to represent the geometry of the printed circuit. It is important to remark that the value of the spatial images are only calculated when the source is placed at the center of each discretization cell. Using this one point integration rule, the entire MoM matrix can be recovered by the following straightforward expression:

$$
\begin{aligned}
Z_{m n}= & Z_{\text {Source }}+Z_{\text {Images }} \\
= & Z_{\text {Source }}+A_{m} A_{n} \vec{F}_{m}\left(\vec{r}_{\rho_{m}}\right) \cdot \overline{\bar{G}}_{\text {Images }}\left(\vec{r}_{\rho_{m}}, \vec{r}_{\rho_{n}}^{\prime}\right) \\
& \cdot \vec{F}_{n}\left(\vec{r}_{\rho_{n}}^{\prime}\right)
\end{aligned}
$$

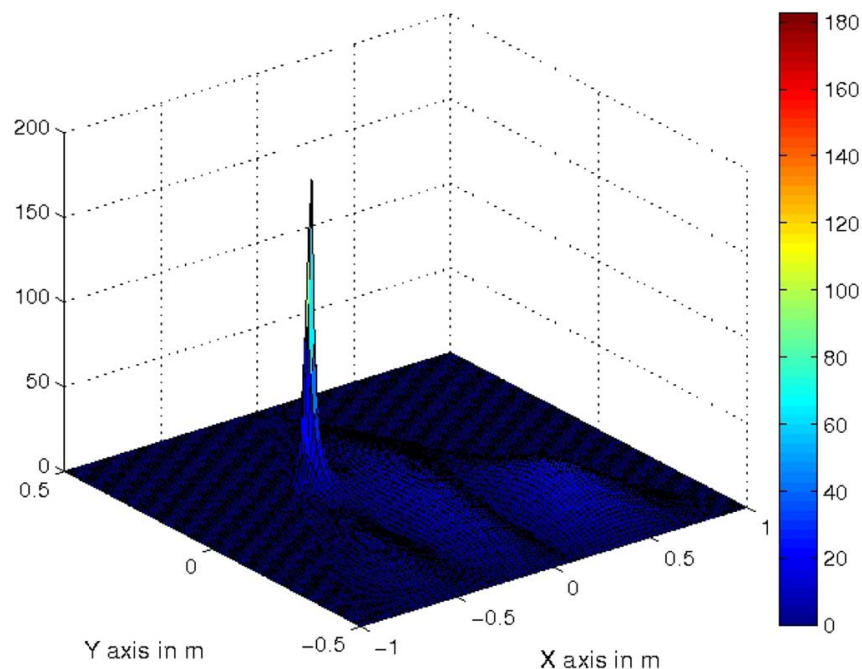

Fig. 12. Electric scalar potential inside the multilayered triangular cavity shown in Fig. 1 when the source is placed at the position $(-0.3 \lambda, 0.13 \lambda, 0.1 \lambda)$.

where $A_{m}$ and $A_{n}$ are the areas of the observation and source cells and $\vec{F}_{m}\left(\vec{r}_{\rho_{m}}\right)$ and $\vec{F}_{n}\left(\vec{r}_{\rho_{n}}^{\prime}\right)$ are the test and basis functions. $\overline{\bar{G}}_{\text {Images }}$ is also the images' contribution to the Green's functions evaluated between the center of the observation cell $\left(\vec{r}_{\rho_{m}}\right)$ and the center of the source cell $\left(\vec{r}_{\rho_{n}}^{\prime}\right)$. The situation when the source is located close to the wall is more complicated. With the new spatial images arrangement presented in this paper, the images adopt a particular disposition as a function of both the source position and the cavity shape, as can be seen in Figs. 2 and 3. In these figures, it can be observed that only one image is actually situated very close to the wall.

For this particular situation, the same procedure explained before is extended. The idea is to extract not only the source term, but also the image which is situated close to the wall (with a distance less than $0.08 \lambda$ ). In this way, the $Z_{\text {source }}$ matrix contains the singular behavior of the source (placed inside the cavity) and the quasi-singular behavior of the images situated close to the wall (and located outside the cavity). It is important to remark that for the integration of this matrix, the values of charges/dipoles associated to the images are not recalculated so the computational cost is still very reduced. In fact, all images values are calculated only once per discretization cell (when the source point is placed at the center of the cell). The value of the extracted image is then reused during the calculation of the $Z_{\text {source }}$ contribution. This approach leads to an efficient MoM implementation, maintaining in all cases good numerical accuracy. To further study the features of the proposed method, we present in Fig. 12 the electric scalar potential inside the multilayered triangular cavity shown in Fig. 1, when the source is situated very close to the wall, at the position $(-0.3 \lambda, 0.13 \lambda, 0.1 \lambda)$. The singular behavior due to the presence of the source can be observed. If we consider only the behavior of the images, the singularity disappears, as is shown in Fig. 13. However, a quasi-singular behavior raises from the image that is situated close the wall (see Fig. 2). Finally, this image is extracted and added to the singular impedance matrix $Z_{\text {source. }}$ In this case, the contribution of reminder to the electric scalar potential is shown 


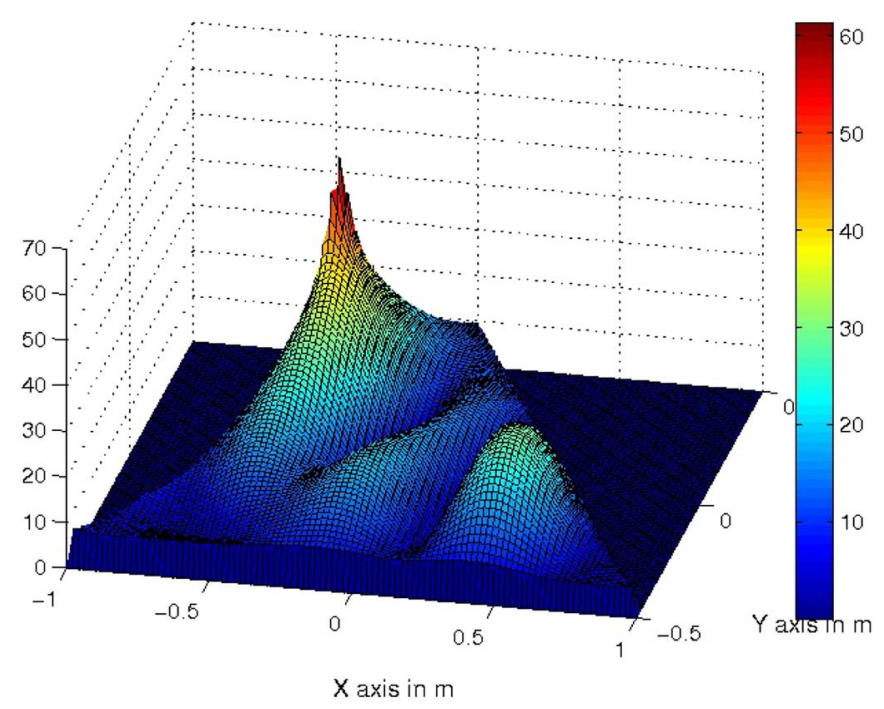

Fig. 13. Images contribution to the electric scalar potential inside the multilayered triangular cavity depicted in Fig. 1 when the source is placed at the position $(-0.3 \lambda, 0.13 \lambda, 0.1 \lambda)$.

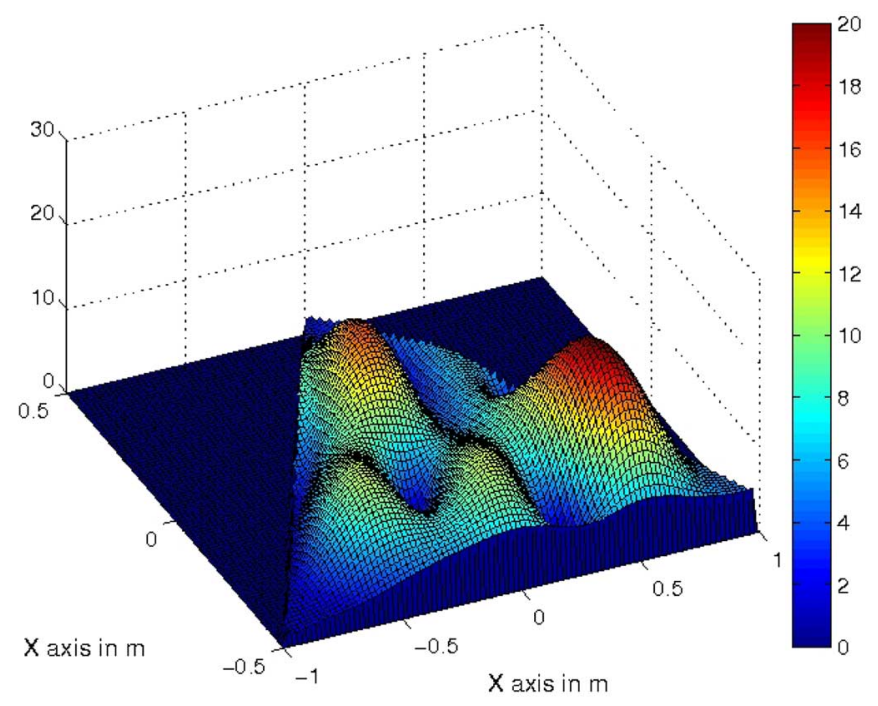

Fig. 14. Nonsingular behavior of the images contribution to the electric scalar potential inside the cavity shown in Fig. 1. The source is placed at the position $(-0.30 \lambda, 0.13 \lambda, 0.1 \lambda)$.

in Fig. 14. This contribution will have a smooth behavior and can be integrated with the one point rule shown in (2).

It is important to point out that, with this one-point rule, only one system of linear equation with $N$ unknowns and two systems with $2 N$ unknowns must be solved for each cell of the printed circuit discretization, leading to a very important reduction in the computational cost needed for the analysis of practical shielded circuits. Moreover, the numerical accuracy obtained with this new MoM implementation is good. In all the examples presented in this paper, the relative errors obtained by the new implementation are below $0.01 \%$, as compared to a traditional MoM implementation.

Finally, note that the procedure described is not a standard extraction of the singular term of the Green's functions, as was done in previous studies [9]. Traditionally, the singular behavior of the Green's functions is treated by extracting the asymptotic

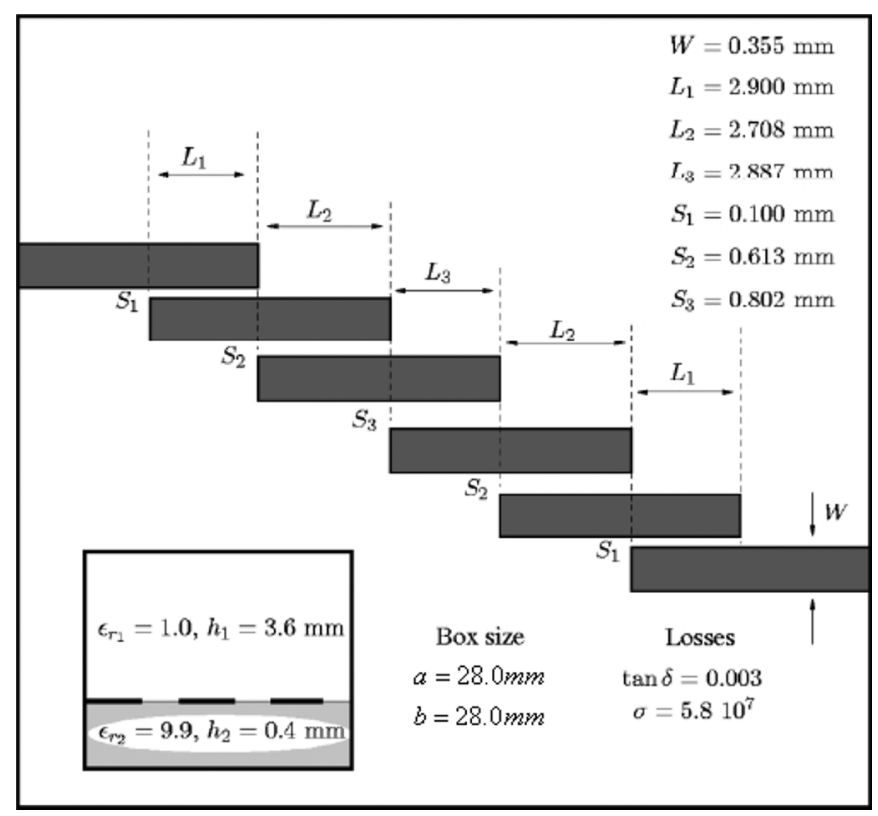

Fig. 15. Boxed microstrip bandpass filter of fourth order based on coupled line sections.

terms with subsequent analytical evaluations of the associated static integrals [13]. On the contrary, with the spatial images technique, the source is naturally separated from the other contributions. Once the contribution from the images is treated as described in (2), the isolated source term $\left(Z_{\text {Source }}\right)$, which provides the singular behavior, can also be treated using other standard asymptotic techniques [13].

\section{RESULTS}

Here, we present several practical examples in order to demonstrate the usefulness of the spatial images technique when modeling practical multilayered shielded circuits. The new image distribution method and the multiring approach are used to keep high accuracy in the spatial image technique implemented. The computational gain obtained with the new MoM implementation is compared to a standard MoM implementation and to other methods. All of the results shown here were obtained on a Pentium IV computer with $3.06-\mathrm{GHz}$ processor and total RAM memory of 2 GB.

\section{A. Coupled-Line Filter}

The first practical example is a boxed microstrip bandpass filter of fourth order based on coupled line sections presented in [14], which is sketched in Fig. 15. The numerical convergence of the spatial images method in this cavity, for all source positions, is achieved with the new images distribution presented in this paper. For the analysis, 12 images are employed and placed around the cavity at the air-dielectric interface.

Fig. 16 shows via a dashed line the filter response obtained by placing the images at a fixed distance of $(0.5 \lambda)$ from the cavity wall. Results obtained using the new algorithm to find the proper location of the images are also presented. For validation, we compare these results with those obtained with an artificial neural-network approach [15] and with measurements. In all cases, the input/output lines and the printed resonators are 


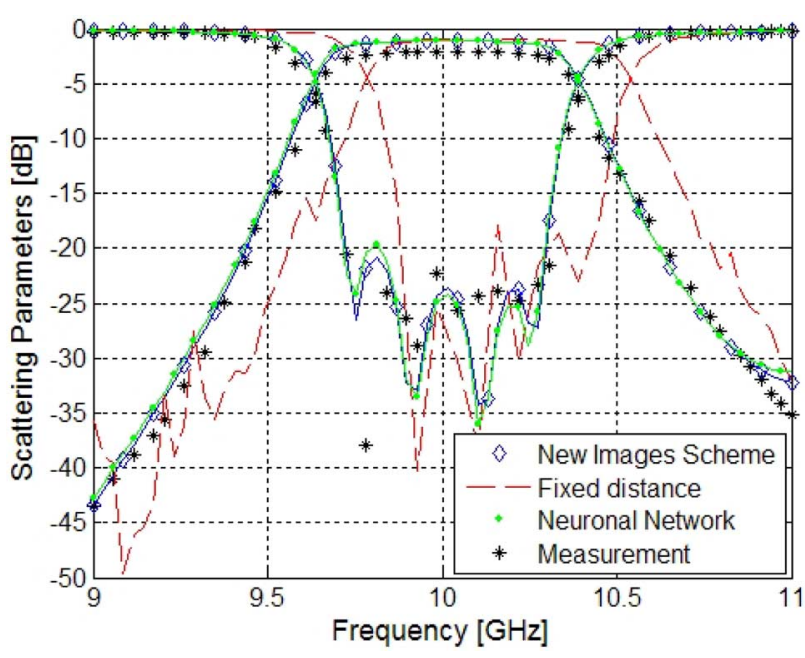

Fig. 16. Results for the bandpass filter shown in Fig. 15, obtained with different techniques. Measured data are also shown as validation.

TABLE I

CPU TIME COMPARISON FOR THE ANALYSIS OF THE FILTER SHOWN IN FIG. 15

\begin{tabular}{|c|c|c|c|}
\hline $\begin{array}{c}\text { Spatial Images } \\
\text { method }\end{array}$ & ADS & $\begin{array}{c}\text { Artificial-Neural } \\
\text {-Network method }\end{array}$ & $\begin{array}{c}\text { Optimized Spatial } \\
\text { Images method }\end{array}$ \\
\hline \hline $48.195(\mathrm{sec})$ & $12.15(\mathrm{sec})$ & $3.2725(\mathrm{sec})$ & $1.2080(\mathrm{sec})$ \\
\hline
\end{tabular}

meshed with a total of 104 rectangular cells. Good agreement is observed when the new algorithm to automatically place the spatial images is used. However, the results obtained when the images are placed at a fix distance show some numerical errors. This is because the input/output printed lines extend close to the cavity walls, and in these regions, the Green's functions exhibit poor convergence rates. However, accurate results are obtained when the new algorithm for image placement is employed. This is because the new algorithm preserve high accuracy, even for source points close to the cavity walls (see Fig. 8).

To show the performance of the new MoM implementation, we compare in Table I the CPU time needed for the analysis of the structure using different approaches. The first one is the spatial images technique using a direct MoM implementation. The second is Agilent Technologies' commercial software Advanced Design System (ADS). The third is the neural network technique derived in [15], and the fourth is the spatial image technique using the new MoM implementation.

It can be seen in the results reported in Table I that the optimized spatial images technique presented in this paper obtains the best computational performance. In particular, the optimized implementation improves the original spatial images technique by a factor of 40, and the commercial software ADS by a factor of 10 . The new technique is even faster than the neural-network method reported in [15] by a factor of 2.7. In addition, the neural-network method needs training time, which can be long. This extra computational effort is not needed when the new spatial images approach is used. To further study the performance of the new technique as compared to the neural-network method reported in [15], we present in Fig. 17 the CPU time per frequency point needed for the analysis of the filter as a function of the number of rectangular cells used in the discretization of the printed lines. Initially, when the number of cells is

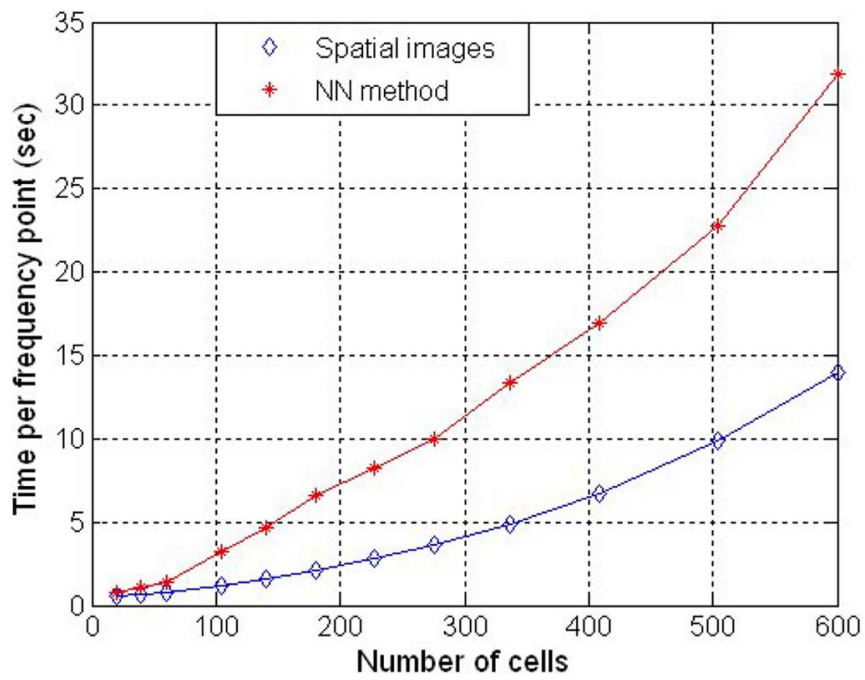

Fig. 17. Time per frequency point (seconds) versus number of cells in the mesh of the circuit.

small, the performances of the novel images technique and of the neural-network method are very similar. However, when the number of cells used in the discretization of the circuit grows, the gain in efficiency of the new spatial images technique begins to be important. This is due to the special treatment of the images contributions using one point integration rule, as reported in (2), and shows the interest of the new technique proposed in this paper.

\section{B. Trapezium-Shaped Cavity Filter}

In this example, we present for the first time a bandpass filter inside a trapezium-shaped cavity, designed with the spatial images technique presented in this paper. The filter follows the transversal topology concept in hybrid printed-waveguide technology introduced in [6]. The key idea is the combination of two different technologies (waveguide and microstrip) to obtain a second-order transversal filter. The structure combines one microstrip printed resonator with a resonance of the trapeziumshaped cavity to build up a second order response. It is important to point out that the modeling of the cavity is a key issue in this structure. This is because the cavity provides one of the resonances of the filter. Consequently, a small error in the modeling of the resonant frequencies of the cavity will lead to a wrong design. The lateral and top views of the filter are shown in Fig. 18. A microstrip printed resonator is placed inside a trapezium-shaped cavity. Printed input/output lines are used to excite the microstrip resonator and one of the resonances of the cavity.

For the design of the structure, the trapezium-shaped cavity is first adjusted to obtain a resonance at the frequency of $4.5 \mathrm{GHz}$. The printed resonator is then optimized to provide the second resonance of the filter. For the analysis of the structure using the spatial images technique, two rings with 20 images are needed in order to obtain a convergent solution in this cavity. The reason to employ two rings (located at heights $z_{1}=1.585 \mathrm{~mm}$ and $z_{2}=3.17 \mathrm{~mm}$ ) is to control and to accurately model the resonance of the cavity. As already stated, this resonance is one of the key elements of the filter (see [6]). After the design process, 

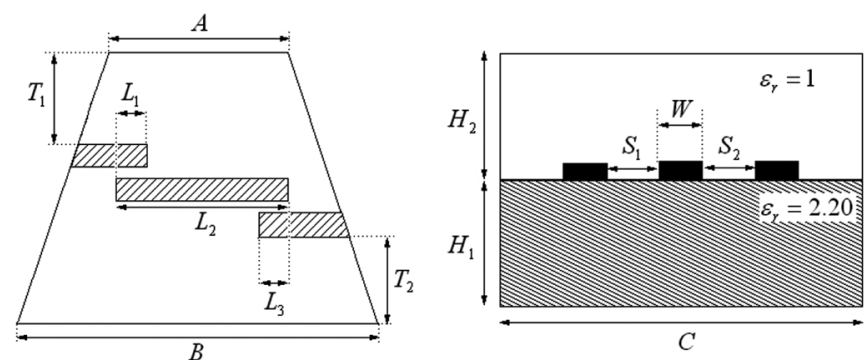

$L_{1}=L_{3}=6 \mathrm{~mm} \quad L_{2}=24 \mathrm{~mm} \quad H_{1}=3.17 \mathrm{~mm} \quad H_{2}=2.83 \mathrm{~mm} \quad A=27.4 \mathrm{~mm}$

$S_{1}=S_{2}=1.5 \mathrm{~mm} \quad T_{1}=T_{2}=15.5 \mathrm{~mm} \quad W=2 \mathrm{~mm} \quad B=56 \mathrm{~mm} \quad C=40 \mathrm{~mm}$

Fig. 18. Novel trapezium-shaped second-order transversal filter.

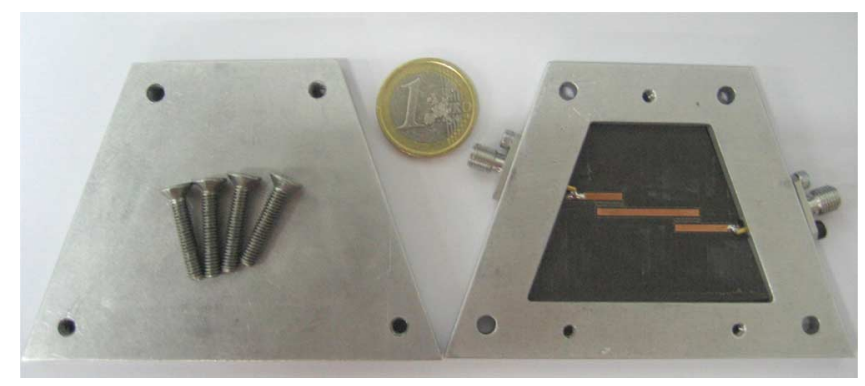

Fig. 19. Aspect of the manufactured breadboard, showing all pieces of the filter.

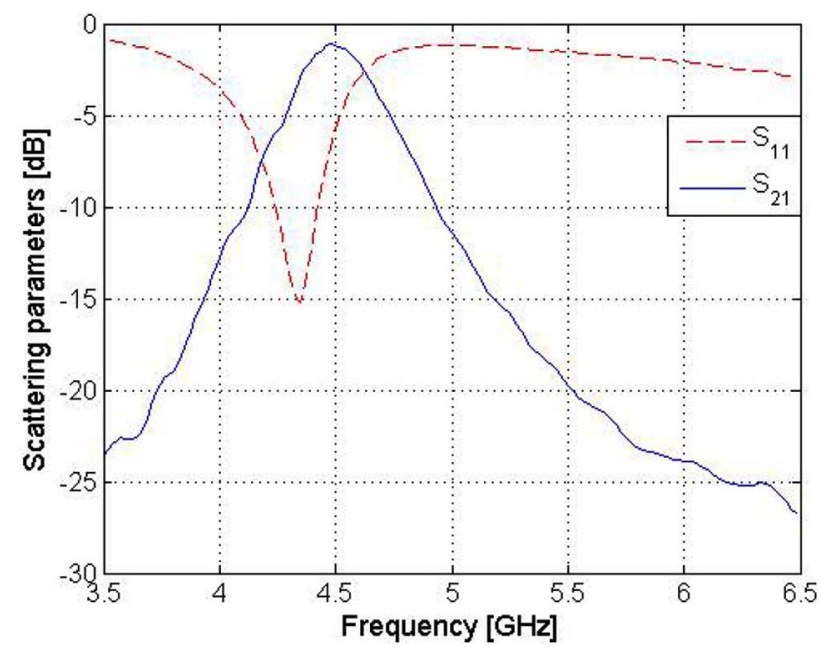

Fig. 20. Measured response for the transversal filter sketched in Fig. 18, obtained when the top cover is removed.

a prototype, shown in Fig. 19, has been manufactured and tested. Fig. 20 presents the measured scattering parameters of the filter when the top cover is removed. In this case, only the microstrip resonance is observed. We can also verify that some of the energy is radiated. This is due to the energy coupled to the mode excited in the cavity, which is radiated through the uncovered top lid. This test shows the importance of the cavity for the proper operation of the filter.

The final filter response is recovered when the trapeziumshaped cavity is closed, as shown in Fig. 21. The two transmission zeros typical of a modified doublet topology can be observed [16]. Good agreement between both measured data and results obtained with the spatial images method is observed. The

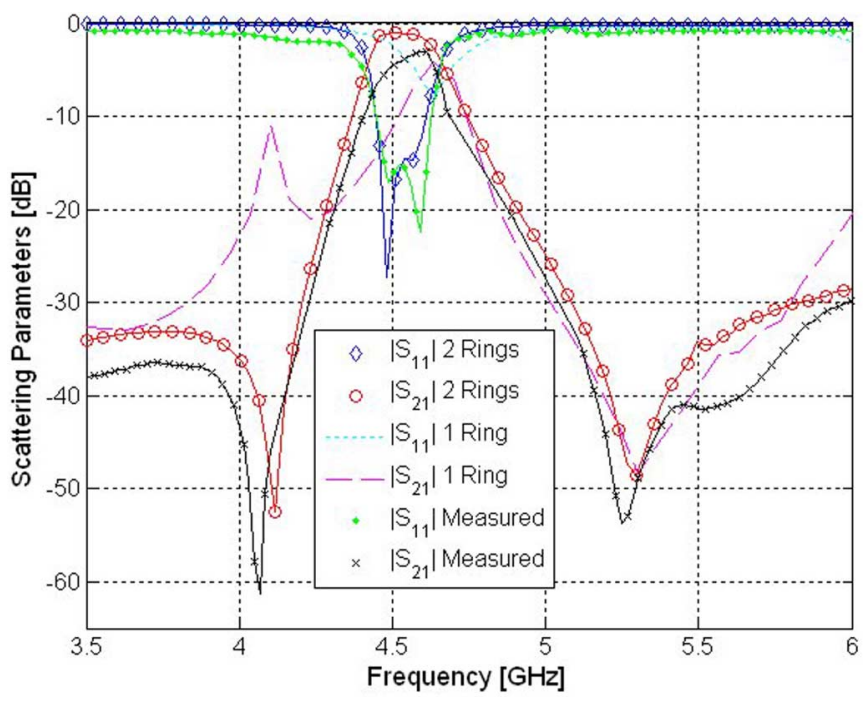

Fig. 21. Results for the bandpass filter depicted in Fig. 18, obtained with one and two rings of images. Losses are included in the dielectric substrate $(\tan \delta=$ $0.004)$ and in the printed metallizations $\left(\sigma=1 \cdot 10^{7} \Omega^{-1} / \mathrm{m}\right)$. Measured data is presented as validation.

TABLE II

CPU TIME COMPARISON FOR THE ANALYSIS OF THE STRUCTURE PRESENTED IN FIG. 18

\begin{tabular}{|c|c|}
\hline $\begin{array}{c}\text { Spatial Images } \\
\text { method }(\mathrm{sec})\end{array}$ & $\begin{array}{c}\text { Optimized Spatial } \\
\text { Images method }(\mathrm{sec})\end{array}$ \\
\hline 121.150 & 5.103 \\
\hline
\end{tabular}

differences in the minimum insertion loss observed within the passband are mainly due to the cavity losses, which are not considered by the developed spatial images technique. In this case, these losses can be important since the filter operates with one of the resonances excited in the partially filled cavity. It is important to remark the essential influence of the trapezium-shaped cavity, which provides one of the resonances of the filter. This influence is correctly modeled by the spatial images technique.

It is worth mentioning that two rings of images are needed to obtain accurate results for this structure due to the presence of the resonant mode of the cavity. Fig. 21 clearly shows that only one ring placed at the dielectric interface $(z=3.17 \mathrm{~mm})$ cannot properly control the resonant mode, leading to incorrect results. However, when a second ring of images is placed at $z=$ $1.585 \mathrm{~mm}$, the response of the filter is correctly recovered. This example shows the practical value of the multiring approach proposed in this paper.

In this example, the microstrip lines were meshed with 150 rectangular cells. Table II shows the CPU time needed for the analysis using a direct MoM implementation and using the optimized spatial images method. It can be seen that the optimized technique presented in this paper really improves the performance of a standard MoM implementation. In this case, no CPU time is given for ADS since this software can only treat rectangular cavities.

\section{Broadside Coupled Filter}

Other interesting examples are broadside-coupled structures used for the design of high-performance microwave filters. 


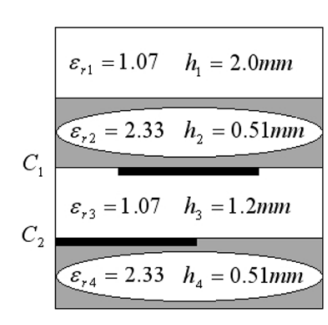

$$
\begin{aligned}
& L_{1}=33.94 \mathrm{~mm} \\
& L_{2}=33.30 \mathrm{~mm} \\
& S_{1}=0.88 \mathrm{~mm} \\
& S_{2}=0.64 \mathrm{~mm} \\
& W=1.49 \mathrm{~mm}
\end{aligned}
$$
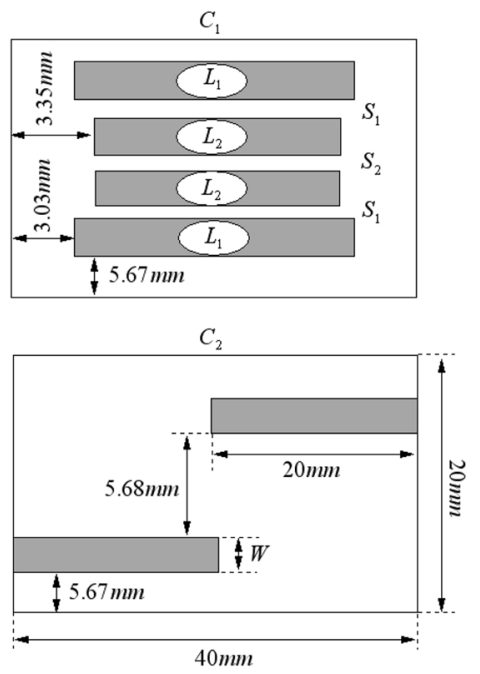

Fig. 22. Bandpass broadside-coupled filter.

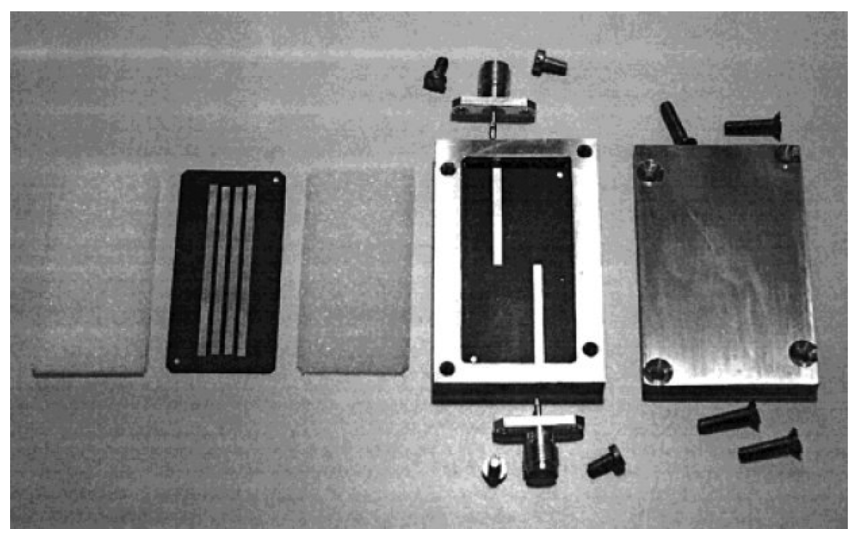

Fig. 23. Aspect of the manufactured breadboard, showing all pieces of the filter.

These types of filters allow the introduction of cross couplings between nonadjacent resonators. The cross couplings can then be used to implement transmission zeros that can significantly increase the selectivity of the filters. A four-pole broadside-coupled filter, depicted in Fig. 22, is proposed to demonstrate the capability of the spatial images technique to analyze these types of broadside-coupled multilayered structures.

To analyze this filter, two rings with 14 images are needed to obtain good convergence. The rings are placed at the first and second air-dielectric interfaces (see Fig. 22). In Fig. 23, a manufactured filter prototype is presented. Simulated versus measured results are included in Fig. 24, showing good agreement.

To perform this analysis, the microstrip lines are meshed with 150 cells. Table III shows the CPU time needed for the analysis of the structure using the new technique and the commercial software ADS. For comparison of the new technique, in this table we give the CPU time when a standard MoM implementation is used, and when the novel implementation presented in this paper is combined with the spatial-domain formulation. We observe a good performance of the optimized spatial technique as compared to both a direct MoM implementation and ADS software.

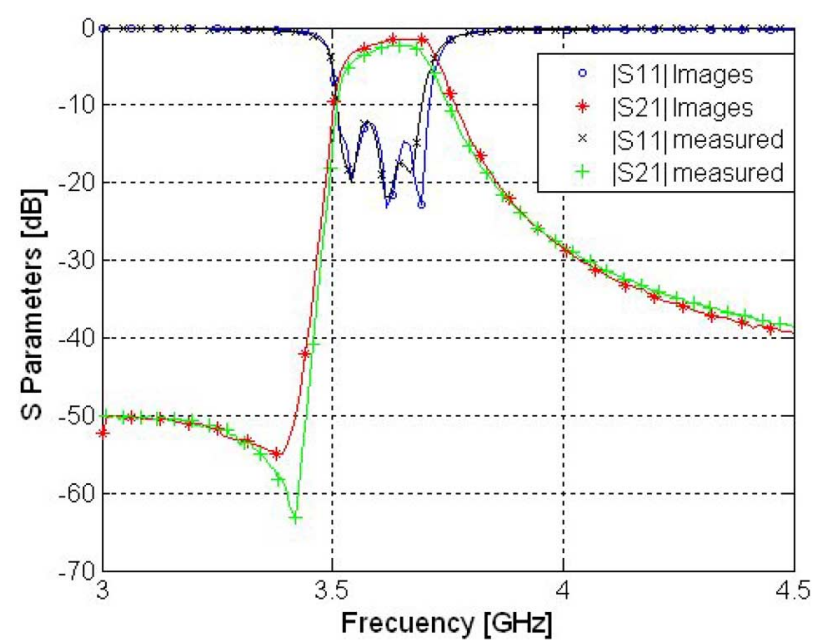

Fig. 24. Results for the bandpass filter shown in Fig. 22. Losses are included in the dielectric substrate $(\tan \delta=0.004)$ and in the printed metallizations $\left(\sigma=1 \cdot 10^{7} \Omega^{-1} / \mathrm{m}\right)$. Measured results are also shown for validation.

TABLE III

CPU TIME COMPARISON FOR THE ANALYSIS OF THE STRUCTURE SHOWN IN FIG. 22

\begin{tabular}{|c|c|c|}
\hline $\begin{array}{c}\text { Spatial Images } \\
\text { method }(\mathrm{sec})\end{array}$ & $\begin{array}{c}\text { ADS } \\
(\mathrm{sec})\end{array}$ & $\begin{array}{c}\text { Optimized Spatial } \\
\text { Images method }(\mathrm{sec})\end{array}$ \\
\hline 87.7103 & 16.25 & 4.9802 \\
\hline
\end{tabular}

\section{CONCLUSIONS}

In this paper, we have presented a practical implementation of the spatial images method for the analysis of shielded multilayered printed circuits. The Sommerfeld transformation is employed to impose the boundary conditions at the top and bottom covers and to take into account for the presence of dielectric layers. A new images arrangement has been proposed in order to calculate the Green's functions with high precision for all positions of the source point. Electrically long cavities are analyzed with a multiring approach, imposing the boundary conditions at different discrete heights. Furthermore, the special features of the spatial images formulation have been exploited to propose a new efficient MoM implementation for the analysis of practical multilayered printed shielded circuits. Several implementation examples with CPU time comparisons have been presented, showing the accuracy and efficiency of the method.

\section{REFERENCES}

[1] R. F. Harrington, Field Computation by Moment Methods. New York: IEEE Press, 1968.

[2] F. Q. Pereira, P. V. Castejon, D. C. Rebenaque, J. P. Garcia, and A. A. Melcon, "Numerical evaluation of the Green's functions for cylindrical enclosures," IEEE Trans. Microw. Theory Tech., vol. 53, no. 1, pp. 94-105, Jan. 2005.

[3] J. S. Gómez-Díaz, M. Martínez-Mendoza, F. D. Quesada-Pereira, J. Pascual-Garcia, and A. Alvarez-Melcon, "Numerical evaluation of the Green's functions for arbitrarily shaped enclosures," in IEEE MTT-S Int. Microw. Symp. Dig., Honolulu, HI, Jun. 6-11, 2007, pp. 1947-1950.

[4] K. A. Michalski and J. R. Mosig, "Multilayered media Green's functions in integral equation formulations," IEEE Trans. Antennas Propag., vol. 45, no. 3, pp. 508-519, Mar. 1997.

[5] K. Michalski, "Extrapolation methods for Sommerfeld integral tails," IEEE Trans. Antennas Propag., vol. 46, no. 10, pp. 1405-1418, Oct. 1998. 
[6] M. Martínez-Mendoza, J. S. Gómez-Díaz, D. C. Rebenaque, J. L. Gómez-Tornero, and A. Alvarez-Melcon, "Design of a bandpass transversal filter employing a novel hybrid waveguide-printed structure," in IEEE MTT-S Int. Microw. Symp. Dig., Honolulu, HI, Jun. 6-11, 2007, pp. 1281-1284.

[7] G. Pelosi and P. Y. Ufimtsev, "The impedance boundary condition," IEEE Antennas Propag. Mag., vol. 38, no. 1, pp. 31-35, Feb. 1996.

[8] C. A. Balanis, Advanced Engineering Electromagnetics. New York: Wiley, 1989.

[9] A. A. Melcon and J. R. Mosig, "Two techniques for the efficient numerical calculation of the Green's functions for planar shielded circuits and antennas," IEEE Trans. Microw. Theory Tech., vol. 48, no. 9, pp. 1492-1504, Sep. 2000.

[10] S. M. Rao, D. R. Wilton, and A. W. Glisson, "Electromagnetic scattering by surfaces of arbitrarily shape," IEEE Trans. Antennas Propag., vol. AP-30, no. 5, pp. 409-418, May 1982.

[11] R. Cools, "Monomial cubature rules since Stroud: A compilation, part 2," J. Comput. Appl. Math, vol. 112, no. 1-2, pp. 21-27, 1999.

[12] J. R. Mosig, Integral Equation Technique. New York: Wiley, 1989.

[13] P. Arcioni, M. Bressan, and L. Perregrini, "On the evaluation of the double surface integrals arising in the application of the boundary integral method to 3-D problems," IEEE Trans. Microw. Theory Tech., vol. 45, no. 3, pp. 436-439, Mar. 1997.

[14] A. A. Melcon, J. R. Mosig, and M. Guglielmi, "Efficient cad of boxed microwave circuits based on arbitrary rectangular elements," IEEE Trans. Microw. Theory Tech., vol. 47, no. 7, pp. 1045-1058, Jul. 1999.

[15] J. P. García, F. Quesada-Pereira, D. C. Rebenaque, J. L. G. Tornero, and A. A. Melcón, "A neural-network method for the analysis of multilayered shielded microwave circuits," IEEE Trans. Microw. Theory Tech., vol. 54, no. 1, pp. 309-320, Jan. 2006.

[16] S. Amari and U. Rosenberg, "A universal building block for advanced modular design of microwave filters," IEEE Microw. Wireless Compon. Lett., vol. 13, no. 12, pp. 541-543, Dec. 2003.

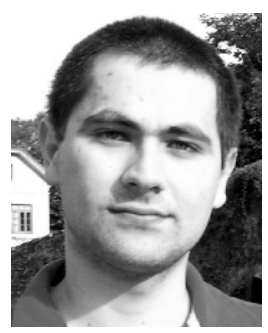

Juan Sebastian Gómez-Díaz (S'07) was born in Ontur (Albacete), Spain, in 1983. He received the Telecommunications Engineer degree (with honors) from the Technical University of Cartagena (UPCT), Cartagena, Spain, in 2006, and is currently working toward the Ph.D. degree at UPCT.

In 2007, he has joined the Telecommunication and Electromagnetic group, UPCT, as a Research Assistant. His current scientific interests include numerical methods and their application in the analysis and design of microwave circuits and antennas.

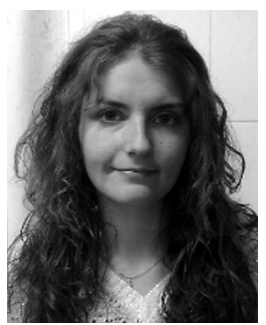

Monica Martínez-Mendoza ( $\mathrm{S}^{\prime}$ 07) was born in Cartagena, Murcia, Spain, in 1983. She received the Telecommunications Engineer degree from the Technical University of Cartagena (UPCT), Cartagena, Spain, in 2006, and is currently working toward the Ph.D. degree at UPCT.

In 2007, she joined the Telecommunications and Electromagnetic Group, UPCT, as a Research Assistant, where she is involved in the development of novel transversal filtering structures for satellite systems. Her current scientific interests include the analysis and design of microwave circuits.

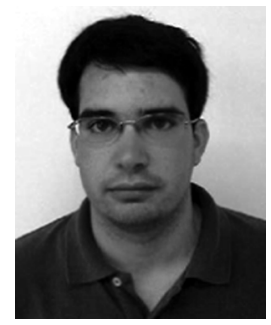

Francisco Javier Pérez-Soler (S'05) was born in Murcia, Spain, in 1981. He received the Telecommunications Engineer degree from the Technical University of Cartagena (UPCT), Cartagena, Spain, in 2004, and is currently working toward the Ph.D. degree at UPCT.

$\mathrm{He}$ is currently with the Communication and Information Technologies Department, UPCT. His current scientific interests include the IE technique for the analysis of antennas and microwave devices.

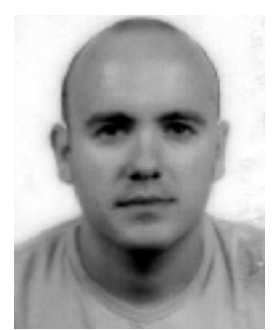

Fernando Quesada-Pereira (S'05-M'07) was born in Murcia, Spain, in 1974. He received the Telecommunications Engineer degree from the Technical University of Valencia (UPV), Valencia, Spain, in 2000, and the Ph.D. degree from the Technical University of Cartagena (UPCT), Cartagena, Spain in 2007.

In 1999, he joined the Radiocommunications Department, UPV, as a Research Assistant, where he was involved in the development of numerical methods for the analysis of anechoic chambers and tag antennas. In 2001, he joined UPCT, initially as an Research Assistant, and then as an Assistant Professor. His current scientific interests include the IE technique applied to the analysis of antennas and microwave devices.

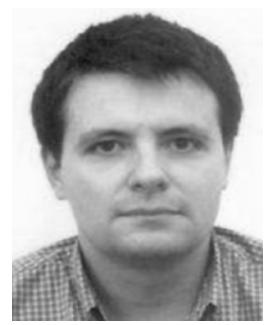

Alejandro Alvarez-Melcón (M'99-SM'07) was born in Madrid, Spain, in 1965. He received the Telecommunications Engineer degree from the Technical University of Madrid (UPM), Madrid, Spain, in 1991, and the Ph.D. degree in electrical engineering from the Swiss Federal Institute of Technology, Lausanne, Switzerland, in 1998.

In 1988, he joined the Signal, Systems and Radiocommunications Department, UPM, as a Research Student, where he was involved in the design, testing, and measurement of broadband spiral antennas for electromagnetic measurements support (EMS) equipment. From 1991 to 1993 , he was with the Radio Frequency Systems Division, European Space Agency (ESA)/European Space Research and Technology Centre (ESTEC), Noordwijk, The Netherlands, where he was involved in the development of analytical and numerical tools for the study of waveguide discontinuities, planar transmission lines, and microwave filters. From 1993 to 1995, he was with the Space Division, Industry Alcatel Espacio, Madrid, Spain, and also with the ESA, where he collaborated on several ESA/ESTEC contracts. From 1995 to 1999 , he was with the Swiss Federal Institute of Technology, École Polytechnique Fédérale de Lausanne (EPFL), Lausanne, Switzerland, where he was involved in the field of microstrip antennas and printed circuits for space applications. In 2000, he joined the Technical University of Cartagena (UPCT), Cartagena, Spain, where he currently develops his teaching and research activities.

Dr. Alvarez-Melcón was the recipient of the Journée Internationales de Nice Sur les Antennes (JINA) Best Paper Award for the best contribution to the JINA'98 International Symposium on Antennas, and the Colegio Oficial de Ingenieros de Telecomunicación (COIT/AEIT) Award for the best doctoral thesis in basic information and communication technologies. 